DOI 10.35381/cm.v5i1.265

\title{
La lógica difusa como herramienta para la evaluación del desempeño de los servidores públicos
}

\section{The Fuzzy logic as a tool for performance evaluation of the public servers}

\author{
Edisson Antonio Carrión Agila \\ ecarriona@psg.ucacue.edu.ec \\ Universidad Católica de Cuenca, Cuenca \\ Ecuador \\ https://orcid.org/0000-0002-7674-3762 \\ Juan Carlos Erazo Álvarez \\ jcerazo@ucacue.edu.ec \\ Universidad Católica de Cuenca, Cuenca \\ Ecuador \\ https://orcid.org/0000-0001-6480-2270 \\ Cecilia Ivonne Narváez Zurita \\ inarvaez@ucacue.edu.ec \\ Universidad Católica de Cuenca, Cuenca \\ Ecuador \\ https://orcid.org/0000-0002-7437-9880 \\ Diego Fernando Trelles Vicuña \\ dtrelles@ucacue.edu.ec \\ Universidad Católica de Cuenca, Cuenca \\ Ecuador \\ https://orcid.org/0000-0002-8466-7165
}

Recibido: 3 de septiembre de 2019

Aprobado: 17 de septiembre de 2019

\section{RESUMEN}

Uno de los elementos de la gestión del talento humano, es la evaluación de desempeño, que, para el caso de los servidores públicos, constituye uno de los temas de mayor importancia en las Instituciones del Estado. La unidad de análisis de la presente 


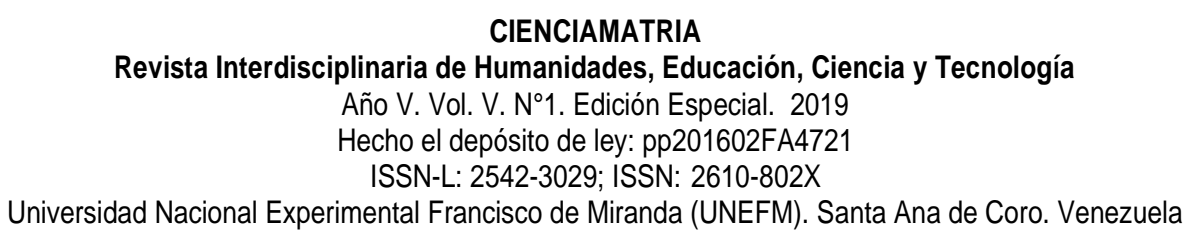

Edisson Antonio Carrión Agila; Juan Carlos Erazo Álvarez; Cecilia Ivonne Narváez Zurita; Diego Fernando Trelles Vicuña

investigación es la Dirección Zonal 6 del SRI en la ciudad de Cuenca, en donde se identificó que existe una afectación en el clima laboral procedente de resultados de evaluaciones de desempeño deficientes. Se propone una metodología de avanzada dentro de la lógica difusa, que es la teoría de los efectos olvidados, a fin de valorar los elementos o variables que inciden en los resultados del proceso de evaluación de desempeño, y que, a pesar de que se tenga un buen sistema de control, siempre existe la posibilidad de no considerar, u olvidar algunas relaciones de causalidad que no siempre resultan explicitas o evidentes, y que provoca que normalmente no sean considerados dentro de este proceso. La investigación fue de tipo no experimental, cuali-cuantitativa, tiene un alcance descriptivo y de finalidad transversal. Como resultado más relevante se determinó que la variable omitida es la elaboración de un óptimo instrumento de evaluación.

Descriptores: Evaluación de desempeño; Lógica difusa; Servicio público; Gestión de talento humano; Competencias.

\begin{abstract}
One of the elements of human talent management is performance evaluation, which, in the case of public employees, constitutes one of the most important issues in State Institutions. The unit of analysis of the present investigation is the Direction Zonal 6 of the $\mathrm{SRI}$ in the city of Cuenca, where it was identified that there is an affectation in the work environment from the results of poor performance evaluations. An advanced methodology is proposed within the fuzzy logic, which is the theory of forgotten effects, in order to assess the elements or variables that affect the results of the performance evaluation process, and that, despite having a good control system, there is always the possibility of not considering, or forgetting some causal relationships that are not always explicit or obvious, and that usually do not consider them in this process. The research was nonexperimental, qualitative-quantitative, has a descriptive scope and a transversal purpose. As a more relevant result, it was determined that the omitted variable is the elaboration of an optimal evaluation instrument.
\end{abstract}

Descriptors: Performance evaluation; Fuzzy logic; Public service; Human resources management; Competences 


\section{INTRODUCCIÓN}

El sector público en el Ecuador ha estado marcado por diversas épocas, que han definido el andar de las instituciones públicas. En este sentido, se puede observar que cada periodo de gestión de un mandatario ha determinado la forma en las que las instituciones de la función ejecutiva han actuado, enmarcados en las normas, leyes, decretos y demás de normativa que delimita el camino por el cual deben actuar las organizaciones del sector público. Dicha normativa establece directrices con el fin de que las instituciones transparenten su gestión y hagan uso adecuado de los recursos del estado, principalmente en la gestión administrativa y financiera.

En este contexto, podemos contrastar dos escenarios en la última década que ha marcado la percepción de los servicios públicos en el país. De acuerdo a la publicación de transparency.org, en el año 2018 el sector público del Ecuador tiene una puntuación de 34/100 en la transparencia de la gestión pública, colocando al país en el puesto 114; contrastando con los resultados del año 2008, el país obtuvo una puntuación de 20/100 y dentro del ranking se ubicó en el puesto 151 (Transparency International, s.f.). En este sentido, la percepción de corrupción en el sector público ha disminuido, principalmente debido a la modernización de los servicios que brinda el estado, así como a las normas que determinan los procesos de gestión administrativa, como es el caso de las normas de gestión de talento humano.

Las Instituciones públicas del estado ecuatoriano están regidas por lo que dictamine y les faculte la ley, reglamentos y demás normas que enmarcan su actuar. Por ejemplo, dentro de la gestión del talento humano, se encuentra dentro del control de la Contraloría General del Estado en los procesos administrativos, el Instituto Ecuatoriano de Seguridad Social, y por el Ministerio de Trabajo (MDT) en todo lo que tiene que ver con la administración de personal. En este sentido, si bien el actuar en la gestión de talento humano de las Unidades Administrativas de Talento Humano de las Instituciones Públicas (UATH) necesariamente se debe enmarcar en lo que la normativa determina, 
existen factores y elementos de los procesos de la Gestión de Talento Humano que pueden ser implementados y gestionados en procedimientos internos de estas instituciones.

Las instituciones de la función ejecutiva del estado, en su mayoría tienen desconcentradas las funciones de cada una de sus actuaciones. Tomando como ejemplo al Servicio de Rentas Internas (SRI), la Dirección Zonal 6 tiene competencia en su actuar sobre las provincias de Azuay, Cañar y Morona Santiago. La sede de la Zonal 6 está ubicada en la ciudad de Cuenca, y a su vez en las dos provincias sobre las cuales tiene competencia, tiene Direcciones Provinciales, con sede en las ciudades de Azogues para la Dirección Provincial de Cañar y Macas para la Dirección Provincial de Morona Santiago.

En este sentido, la dirección general del SRI mediante resolución expresa, delega competencias a los directores zonales con el fin de cumplir con los principios de desconcentración y descentralización contemplados en la constitución de la república, entre estas competencias, se encuentran tema que facilitan la gestión de talento humano. (Servicio de Rentas Internas, 2016)

De acuerdo con lo que afirma el Servicio de Rentas Internas, la institución se ha caracterizado por ser autónoma en la definición de políticas y estrategias de gestión, y como describe esta Institución en su página web, la recaudación de impuestos se ha incrementado en las últimas décadas, y de acuerdo al análisis que realiza el SRI, esto responde a la gestión eficiente de la institución, como es la implementación y mejoramiento de sistemas de alta tecnología, como la Facturación Electrónica, SRI móvil, o los servicios en línea, lo que ha provocado la disminución de costos indirectos de los contribuyentes e impregnando la cultura tributaria en la ciudadanía, y no solo responde a reformas en leyes o normas tributarias. Esto da en evidencia de acuerdo a las cifras publicadas por el SRI, que el periodo 2000 y 2006 la recaudación casi alcanzó los 22.000 millones; entre tanto que en los periodos de los años 2007 y 2013 la recaudación superó los 60.000 millones de dólares. Dichos valores publicados. (Servicio de Rentas Internas, 


\section{CIENCIAMATRIA}

Revista Interdisciplinaria de Humanidades, Educación, Ciencia y Tecnología

Año V. Vol. V. №1. Edición Especial. 2019

Hecho el depósito de ley: pp201602FA4721

ISSN-L: 2542-3029; ISSN: 2610-802X

Universidad Nacional Experimental Francisco de Miranda (UNEFM). Santa Ana de Coro. Venezuela

Edisson Antonio Carrión Agila; Juan Carlos Erazo Álvarez; Cecilia Ivonne Narváez Zurita; Diego Fernando Trelles Vicuña

\section{s.f.)}

En cuanto a la gestión de talento humano que se aplica en las instituciones del estado ecuatoriano, las mismas que están basadas en lo que manda y describe la LOSEP, específicamente en lo que se denomina el "sistema integrado de desarrollo del talento humano del sector público", se describe en el artículo 53:

Es el conjunto de políticas, normas, métodos y procedimientos orientados a validar e impulsar las habilidades, conocimientos, garantías y derechos de las y los servidores públicos con el fin de desarrollar su potencial y promover la eficiencia, eficacia, oportunidad, interculturalidad, igualdad y la no discriminación en el servicio público para cumplir con los preceptos de esta Ley. (Asamblea Nacional Constituyente, 2010)

En este contexto, el mismo cuerpo legal describe 5 subsistemas, los mismos que se detallan a continuación:

Figura 1.

Descripción de los subsistemas de talento humano en el sector público

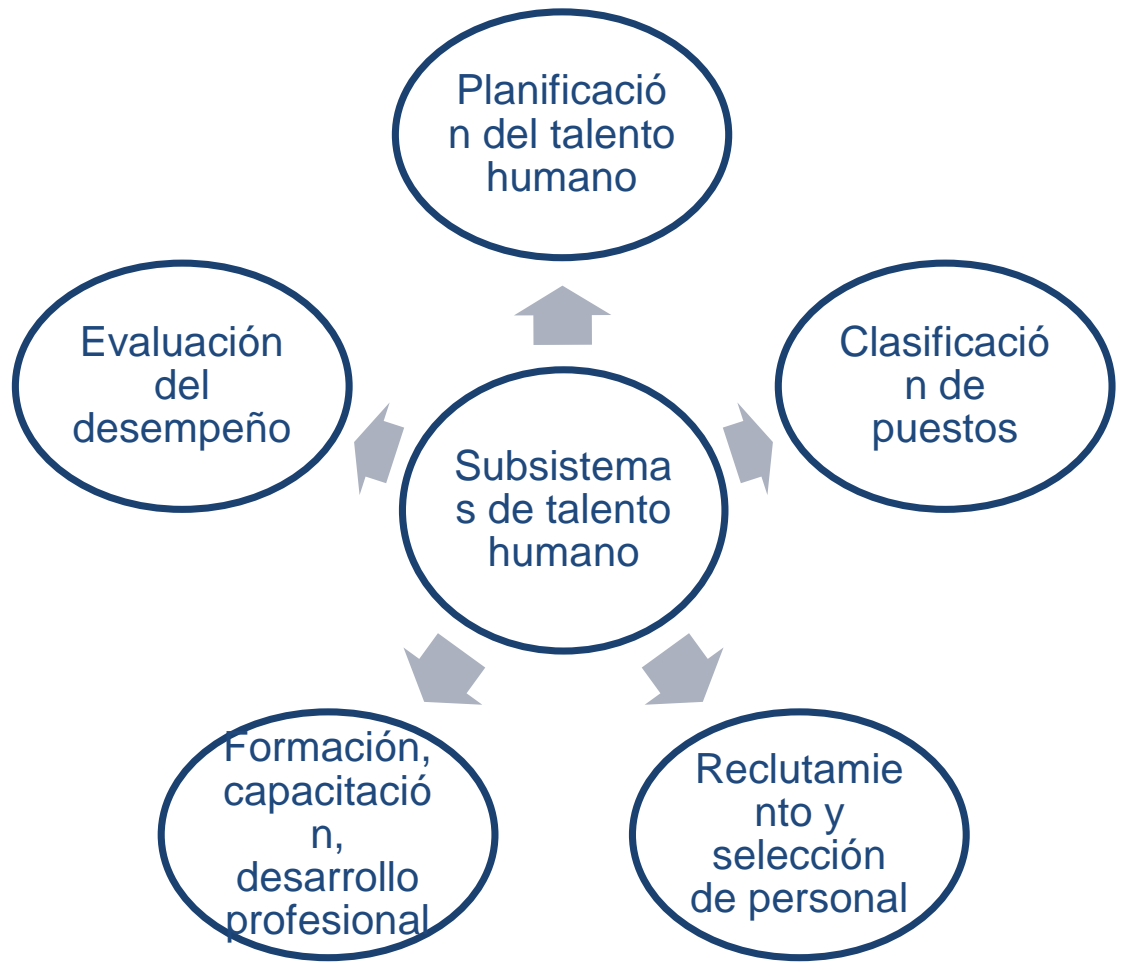

Fuente: Asamblea Nacional Constituyente, 2010 
Entre uno de los subsistemas o elementos de la gestión del talento humano, se encuentra la evaluación de desempeño, que, para el caso de los servidores públicos, constituye uno de los temas de mayor importancia en las Instituciones de Estado, respondiendo a la necesidad de mejorar la calidad de los servicios públicos brindados a la ciudadanía, además de las exigencias del marco legal y normativo de la función ejecutiva.

En este contexto, la Institución tiene implementado un procedimiento interno para controlar u mejorar la ejecución de este proceso. Sin embargo, a pesar de tener procedimientos y directrices, en el proceso es posible que se enfrentes fallas 0 dificultades, siendo una de las principales la deficiente o nula retroalimentación por parte del jefe inmediato, la misma que debe ser efectuada durante todo el periodo a ser evaluado, con el fin de establecer planes de mejora continua oportunos que aseguren un desempeño laboral efectivo y eficiente, evitando así las consecuencias y afectaciones al servidor como la estabilidad laboral, generación de riesgos psicosociales y principalmente, afectando al clima laboral de la unidad y de la Institución, todos procedentes de una calificación de evaluación de desempeño regular o deficiente.

En la presente investigación, se propone una herramienta de avanzada que ofrece la lógica difusa, Kaufmann y Gil Aluja desarrollan las teorías del expertizaje con el objetivo de tratar de acotar la incertidumbre dentro de un problema, a través de variables, elemento o factores omitidas por los expertos (Kaufmann y Gil-Albuja, 1989), que para este caso, son los gestores dentro del proceso de evaluación de desempeño de una Institución Pública, y que deberán ser consideradas por los servidores públicos que ejercen el rol de evaluadores, con la finalidad de mejorar los resultados de las evaluaciones de desempeño y disminuir los efectos negativos de una mala evaluación, en específico al clima laboral. 


\section{LA EVALUACIÓN DE DESEMPEÑO POR COMPETENCIAS}

Alles describe que la competencia es una característica presente en las personas, que está relacionada de alguna manera, en algunos casos innata, con un estándar de efectividad o con la forma en de desarrolla una actividad o trabajo, que, para algunos casos, es superior a otros, definiendo las competencias adquiridas o innatas para desarrollar un puesto de trabajo.

Sin embargo, los gestores de talento humano solamente deben interesarse en aquellas características (que serían las competencias) que hagan eficaces a las personas dentro de la organización, y potenciarlas mediante el desarrollo o adquisición (mediante formación o capacitación) de las mismas. (Alles, 2013). En este sentido, una discusión que ha tomado fuerza en las últimas décadas en las organizaciones es la medición de la competencia, considerando que las empresas en los últimos tiempos invierten por el desarrollo del capital humano (Soler y Castillo, 2009).

Con el antecede de haber definido lo que es una competencia, lo siguiente es medirla. En este sentido, una discusión que ha tomado fuerza en las últimas décadas en las organizaciones es la medición de la competencia, considerando que las empresas en los últimos tiempos invierten por el desarrollo del capital humano, y es en este momento cuando salta a la escena la evaluación de desempeño (Soler y Castillo, 2009).

Para Alles, en la evaluación de desempeño hay tres puntos de importancia:

- Definición del cargo: garantizar que el evaluador y el evaluado, conozcan claramente los niveles e indicadores esperados para el cargo, y que estén de acuerdo con las responsabilidades y los criterios de evaluación.

- Evaluar el desempeño: proceso que debe desarrollarse en función del cargo y las competencias previamente definidas, utilizando una métrica (como escala) que debe estar definida y socializada previamente.

- Retroalimentación: generar un ambiente de tipo entrevista, en el cual el evaluador comente acerca del desempeño del evaluado, el mismo que debe ser continuo durante todo el proceso. 
Así mismo la autora indica que el análisis de la gestión o el desempeño de una persona tienen a su vez tres momentos diferentes:

1. Fijar objetivos, es una etapa inicial en donde se determina que se espera como resultados 0 productos entregables del cargo y las competencias 0 comportamientos esperados y prioritarios para el pedido. Dicho proceso debe ser elaborado mediante reunión entre evaluador y evaluado y de ser posible dejar sentado en acta.

2. Evaluación del progreso. Recomienda mantener reuniones periódicas de progreso antes de culminar el periodo de evaluación, que generalmente es de un año, con el fin de revisar logros y avances de objetivos, en las cuales se establezca el grado de cumplimiento de factores (en relación con el comportamiento o las competencias) o competencias, según el método de evaluación elegido.

3. Cumplido el periodo de evaluación. Elaborar una retroalimentación final de los resultados obtenidos por el evaluado. Se precisa que si el colaborador tuvo dos dependencias, será evaluada por ambos jefes. (Alles, 2008)

EI MDT ejecuta anualmente el proceso de evaluación de desempeño a través de la UATH de cada Institución, basándose en la norma técnica del subsistema de evaluación de desempeño y en lo que dictamina la Ley Orgánica de Servicio Público (LOSEP). Al revisar por otro lado lo que dispone la norma técnica del subsistema de selección de personal, se desprende que, en los procesos de selección del sector público, se utiliza la gestión por competencias, dado que, para ser declarado como ganador de un concurso de méritos y oposición, se debe cumplir con todos los requisitos, entre los cuales está la medición de las competencias técnicas y psicométricas para el cargo. (Ministerio del Trabajo, 2019)

En este sentido, la evaluación de desempeño en el sector público, propone un modelo para la evaluación de desempeño, que contempla dos grandes y principales enfoques: 
evaluación del desempeño individual que mide los logros y capacidades individuales, y evaluación del desempeño organizacional, la que mide la contribución de los servidores públicos, la satisfacción usuarios internos y externos, y los objetivos institucionales y de procesos. Sin embargo, es importante destacar un factor adicional, que es castigador, es decir, no suma a la ponderación total de la evaluación de desempeño, sino que su incumplimiento disminuye puntuación. (Ministerio del Trabajo, 2018)

Figura 1.

Factores de evaluación de desempeño

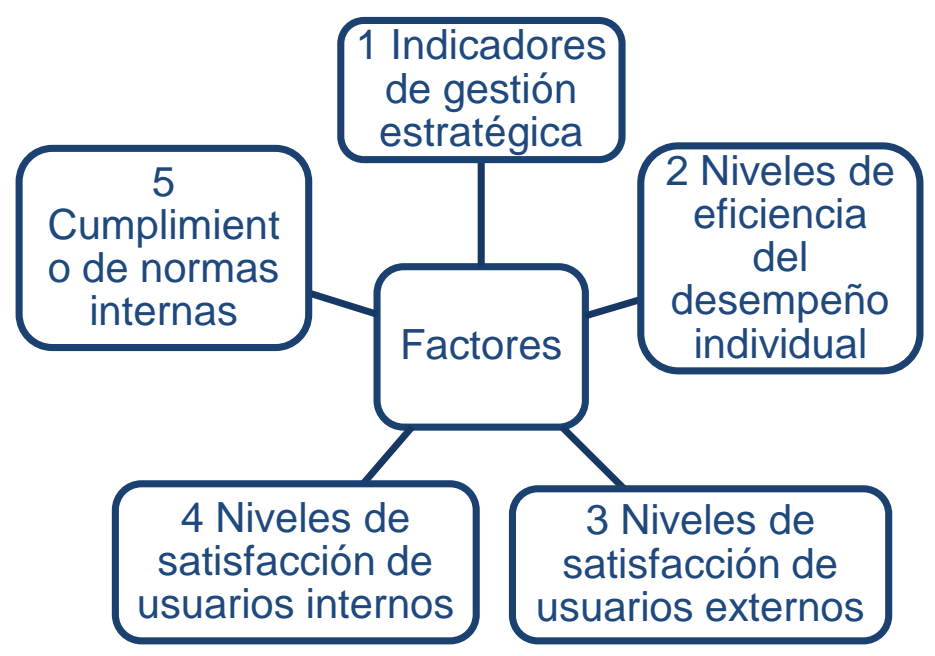

Fuente: Ministerio del Trabajo 2018

Contrastando esta metodología con lo planteado por Alles, se observa que, para el caso de estudio, esto es el SRI, se aplica un modelo de evaluación de desempeño de $270^{\circ}$. Por otro lado, una vez concluido el proceso de evaluación, se tienen los efectos de los resultados, siendo los más preocupantes, los resultados de regular e insuficiente, que para el primer caso ocurre cuando se tiene una calificación entre $70 \%$ y $79,99 \%$, teniendo como efecto la ejecución de una nueva evaluación de desempeño del servidor en el lapso de 3 meses, y si el resultado es nuevamente de regular o insuficiente, se procede a la desvinculación del servidor; a su vez, si el resultado es de insuficiente, esto es con una 
igual o inferior a 69,99\%, se procede a una nueva evaluación al servidor en un periodo de 2 meses, y si el resultado es de regular, se procede con lo indicado en el caso anterior, y si es de insuficiente, se procede a la desvinculación del servidor. (Ministerio del Trabajo, 2018)

Como es de suponer, en el caso de servidores que se enmarquen en una de estas dos situaciones $<<$ resultados de regular o insuficiente $>>$, se afecta directamente el clima laboral, dados los roces entre evaluador y evaluado, así como por la afectación de la estabilidad laboral.

\section{LA LÓGICA DIFUSA COMO HERRAMIENTA DE EVALUACIÓN}

La creación de la lógica difusa remonta a la década de los años sesenta, y se le atribuye a Lotfi Zadeh, dada su publicación "Fuzzy Sets" presentando planteamientos matemáticos que encajan en la teoría de subconjuntos borrosos (Zadeh, 1965), con el paso del tiempo ha tomado fuerza en diversos campos disciplinarios, principalmente en el control de procesos empresariales, en las tecnologías de la información y computación y numerosas aplicaciones en la economía.

La teoría fundamenta los conjuntos de números difusos o borrosos, que representan la pertenencia de elementos o grupos de elementos a una categoría o clasificación, pertenencia que se expresa con intensidades o grados diversos de acuerdo con opiniones de expertos, lo que implica que existan múltiples niveles de pertenencia entre los valores extremos de cada intervalo, incluso con la oportunidad de establecer referencias de parecido entre los límites y sus matices internos (Pérez y León, 2007) Referenciando el tema de análisis de la presente investigación, esto es la evaluación de desempeño, se encuentran variados estudios acerca de este proceso, como es el modelo producido por Payán y Luhrs (2013), en donde se menciona la inferencia difusa para el análisis de programas sociales; se tiene el diseño de evaluación de competencias 
comunes de Manjarrés, Castell y Luna (2013), las mismas que se estudiaron en relación con determinado tipo de empleados, usando a la lógica difusa en el análisis de resultados. Por lo que, como es de suponer, estas afirmaciones dispersan lo que la lógica tradicional define, es decir, que solo existe "lo blanco o lo negro", sino que abre un abanico amplio de escalas de grises entre estos extremos, es decir, los grados de pertenencia, por lo tanto, la lógica difusa representa con mayor ajuste la realidad de los fenómenos sociales o de las variables analizadas (Vidal Beltrán, Erazo Álvarez \& Narváez Zurita, 2019).

\section{FUNCIONES DE PERTENENCIA A PARTIR DE EXPERTOS}

Una de las maneras con la cual se puede determinar el grado o nivel de pertenencia de una variable dentro de un conjunto borroso, y la que es utilizada en la presente investigación, es el expertizaje y contraexpertizaje, dado que permiten valorar las opiniones de diversos expertos sobre una variable y su incidencia en un proceso determinado (Kaufmann y Gil, 1993).

El proceso de expertizaje se basa en obtener las opiniones de los expertos acerca de la incidencia de ciertas variables en un procedimiento o en un fenómeno, para luego vaciarlas en una matriz de incidencia que establece una banda global en donde se agrupa todos los valores límites dados para cada fenómeno o efecto. Para este caso, y a partir de las opiniones dadas por los servidores evaluadores de la Institución y los ejecutores del proceso de evaluación sobre la valoración de factores y/o variables, surgirán las categorías detalladas en los resultados.

El proceso de contraexpertizaje se efectúa luego de lo descrito en el párrafo anterior, es decir luego de realizar el proceso de expertizaje, y básicamente es replicar el procedimiento, pero, se recomienda aplicarlos a distintos expertos y no a los que nos dieron su opinión inicialmente.

Esto desemboca en lo que se denomina expertón, a partir del cual se establecen los nuevos límites para cada categoría, lo que hace caer la entropía y reduce las bandas, es decir, disminuye la incertidumbre. 


\section{LA TEORÍA DE LOS EFECTOS OLVIDADOS}

Una de las herramientas que nos ofrece la lógica difusa, es la teoría de los efectos olvidados, y que fundamenta su procedimiento en que en todo proceder o proceso, existe la posibilidad de no considerar, sea por olvido o de manera involuntaria, variables 0 relaciones de incidencia que en la mayoría de los casos no son visibles o explícitas, situaciones que se presentan a pesar de que se cuente con un mecanismo de control. Estas situaciones se presentan en situaciones donde se analizan efectos sobre efectos, quedando incidencia no visible dada la acumulación de causas que las provocan.

Por naturaleza, las personas necesitan reforzar el análisis con modelos o herramientas capaces de mostrar la relación de causalidad entre variables o factores de fenómenos, situación que está presente en todas las situaciones causa - efecto.

Como es de suponer, en los fenómenos en donde se presentan causalidades de mamera encadenada, es en donde más el ser humano es propenso a olvidar u omitir variables que resultarían importantes en los procesos. Por lo tanto, cada olvido lleva como consecuencia efectos secundarios que van repercutiendo en todas las relaciones de incidencia en fenómeno determinado. (Gil y Barcellos, 2010)

\section{LA EVALUACIÓN DE DESEMPEÑO USANDO TÉCNICAS DIFUSAS}

Rico y Tinto describen que una de las aplicaciones posibles de la matemática borrosa es en el proceso de selección de personal para las organizaciones (Rico y Tinto, 2009), lo que también puede aplicarse dentro de las evaluaciones de desempeño por competencias.

Para Alles, el analizar el desempeño de un colaborador es un instrumento para liderar y controlar el cumplimiento de indicadores del personal. Alles señala que uno de los objetivos fundamentales de este proceso es el desarrollo profesional, y en ocasiones el personal, de los trabajadores, así como la mejora permanente de resultados de la empresa a través del aprovechamiento adecuado del talento humano. (Alles, 2008) 
Se puede por lo tanto deducir que es una necesidad imperiosa de toda organización poder medir la brecha existente entre la competencia (o variable) ideal determinada en el perfil del cargo y el grado de competencia que tiene el servidor o colaborador.

Soler y Castillo en su modelo de evaluación de competencias, en donde abordan la herramienta de la lógica difusa, explican que, para poder calcular la evaluación de los diferentes perfiles, se debe definir un intervalo de confianza $(\mu)$, en el cual se enmarcan los criterios de evaluación, en un intervalo de 0 a 1, [0, 1]. (Soler y Castillo, 2009)

En este sentido, tal como se mencionaba anteriormente, una de las herramientas de avanzada dentro de la lógica difusa es la teoría de los efectos olvidados. Diversos autores han aportado valiosas investigaciones que demuestran la efectividad de esta herramienta para disminuir la incertidumbre en la gestión empresarial, entre aquellos: Luna, Tinto, Sarmiento y Cisneros (2017) abordan las distintas acciones mediante la utilización de teoría del expertizaje, volcando toda esta información en una matriz de efectos olvidados que permita tomar decisiones.

Tinto, Luna y Cisneros (2017) abordan la teoría de efectos olvidados determinando y analizando variables ocultas que no son detectadas y que afectan al proceso de comercialización de calzado en el cantón Gualaceo de la provincia del Azuay.

Rico y Tinto (2010), que plantean la utilización de herramientas basadas en la teoría de los subconjuntos difusos, entre aquellos el expertizaje y el contraexpertizaje, así como la teoría de los efectos olvidados en el tratamiento posterior de información con la finalidad de mejorar la toma de decisiones, o tomar las decisiones adecuadas a mediano y largo plazo. Los autores Gento, Lazzari y Machado (2001) expresan los resultados de esta metodología en distintos problemas de la gestión empresarial, en donde establecen la persistencia estricta y no estricta de una matriz de incidencia.

\section{METODOLOGÍA}

El presente trabajo de investigación es de tipo no experimental, considerando que se estudia la naturaleza de la Institución en su estado actual, para posteriormente analizarlos 
y explicarlos (Cerón, Erazo, Ormaza, y Narváez, 2019). Así mismo es cualicuantitativa, ya que en un principio se tuvo una postura cualitativa, sin embargo, en el transcurso de la investigación, se utilizaron métodos estadísticos para el análisis de resultados, por lo que se tornó en una investigación mixta (Cordero, Narváez, y Erazo, 2019), mayoritariamente cualitativa.

Esta investigación tiene un alcance descriptivo, dado que se caracterizó una realidad de la institución en referencia a la evaluación de desempeño y su procedimiento en la Institución. En cuanto a la finalidad, el trabajo de investigación tomó una postura transversal, dado que se obtienen y analizan los datos en un momento, y no a través del tiempo.

Por otro lado, en cuanto a los métodos, el histórico-lógico está plasmado en el marco teórico del presente trabajo de investigación, dado que se describieron y analizaron diversas teorías y modelos para la evaluación de desempeño, y se les dio un orden lógico utilizando la cronología de sus publicaciones. El método analítico-sintético es aplicado también en el marco teórico de este trabajo de investigación, descomponiendo los temas y puntos de cada modelo estudiado, para determinar qué elementos son aplicables, con el fin de proponer el modelo que mejor se adapte a la realidad de la institución. El método inductivo-deductivo fue aplicado para el análisis de los resultados obtenidos de la aplicación de los métodos para recabar la información. Una vez analizados dichos resultados, se establecieron los elementos para la propuesta del modelo de evaluación de desempeño.

En cuanto a las técnicas, se utilizó la encuesta con el fin de levantar toda la información posible para analizar los efectos olvidados, por estos motivos se utilizó el instrumento de cuestionario estructurado.

La presente investigación está enfocada en el proceso de evaluación de desempeño en las Instituciones del sector público, a efectos de valorar los elementos o variables que inciden en los resultados del proceso de evaluación de desempeño en los servidores públicos, y que, a pesar de contar con un mecanismo de control que es bueno, está 
latente la posibilidad omitir, o no considerar algunas relaciones de causalidad que generalmente no son evidentes o explicitas, y que posiblemente provoca que no sean considerados dentro de este proceso, para esto se utilizó las herramientas de la lógica difusa, específicamente la teoría de los efectos olvidados.

\section{RESULTADOS}

Con el propósito de poder llegar a determinar qué efectos o variables son olvidadas dentro del proceso de evaluación de desempeño en la Institución, y mediante la utilización de herramientas de avanzada, se procede a elaborar una matriz de acciones y efectos con el fin de tratar de mejorar los resultados de las evaluaciones de desempeño y su incidencia en el clima laboral de los servidores públicos, concurriendo al conocimiento y experticia de los expertos, que, para este caso de estudio, son los servidores públicos encargados de ejecutar el proceso de evaluación en la Institución, puntualmente al personal de la UATH zonales y nacional del SRI.

La información requerida y sobre la cual se procede el análisis de los expertos, se presenta en la siguiente tabla:

\section{Tabla 1}

\section{Matriz de Acciones y Efectos}

\section{Acciones}

\section{Efectos}

1. Socializar el métodos, instrumentos y A. Disminuir la resistencia a la evaluación factores de evaluación

\begin{tabular}{|c|c|}
\hline $\begin{array}{l}\text { 2. Mantener actualizadas las metodologías y } \\
\text { los criterios de evaluación }\end{array}$ & $\begin{array}{l}\text { B. Elaborar un óptimo instrumento de } \\
\text { evaluación }\end{array}$ \\
\hline 3. Infraestructura tecnológica actualizada & $\begin{array}{l}\text { C. Evitar inconvenientes operativos en la } \\
\text { ejecución del proceso de evaluación }\end{array}$ \\
\hline $\begin{array}{l}\text { 4. Mantener entrevistas regulares de } \\
\text { avances en los procesos para el } \\
\text { cumplimiento de indicadores }\end{array}$ & $\begin{array}{l}\text { D. Evitar criterios de evaluación basados en } \\
\text { una sola impresión }\end{array}$ \\
\hline
\end{tabular}




\section{CIENCIAMATRIA}

Revista Interdisciplinaria de Humanidades, Educación, Ciencia y Tecnología

Año V. Vol. V. NN1. Edición Especial. 2019

Hecho el depósito de ley: pp201602FA4721

ISSN-L: 2542-3029; ISSN: 2610-802X

Universidad Nacional Experimental Francisco de Miranda (UNEFM). Santa Ana de Coro. Venezuela

Edisson Antonio Carrión Agila; Juan Carlos Erazo Álvarez; Cecilia Ivonne Narváez Zurita; Diego Fernando Trelles Vicuña

5. Implementar una bitácora de E. Evitar criterios negativos del evaluador por acontecimientos (negativos y positivos) acontecimientos recientes sobre el cumplimiento de indicadores

$\begin{aligned} & \text { Establecer un mecanismo } \\ & \text { comunicación efectiva }\end{aligned}$
$\begin{aligned} & \text { resultados } \\ & \text { resuances }\end{aligned}$

7. Implementar la aprobación del jefe G. Disminuir la subjetividad del evaluador mediato en la evaluación del servidor

\begin{tabular}{lll}
\hline $\begin{array}{l}\text { 8. Implementar una metodología de } \\
\text { levantamiento continuo de necesidades de } \\
\text { capacitación }\end{array}$ & $\begin{array}{l}\mathrm{H} \text {. Detectar fallas en conocimientos que } \\
\text { impidan la consecución de objetivos y la } \\
\text { incompetencia del evaluado }\end{array}$ \\
$\begin{array}{ll}\text { 9. Mantener actualizados los manuales y } \\
\text { descriptivos de puestos en donde se definan } \\
\text { claramente las competencias }\end{array}$ & $\begin{array}{l}\text { I. Definiciones claras de las competencias } \\
\text { necesarias para el cargo }\end{array}$ \\
\hline $\begin{array}{ll}\text { 10. Socializar los descriptores de cargos con } \\
\text { el personal }\end{array}$ & $\begin{array}{l}\text { J. Posibilidad de identificar mejoras en el } \\
\text { desarrollo de competencias }\end{array}$ \\
\hline
\end{tabular}

En la tabla mostrada anteriormente, se observa una matriz cuadrada, es decir, que contiene el mismo número de variables. Con esto, aplicando dos de las posibilidades que ofrece la lógica difusa, como es el caso la teoría de los efectos olvidados y el proceso de expertizaje, se puede encontrar el efecto o variable olvidada, con el fin de proponer una solución a los resultados regulares e insuficientes que afectan al clima laboral de los servidores públicos, acortando la incertidumbre que facilite la implementación de una metodología o procedimiento en la Institución que mejore estos resultados.

Con este antecedente, Kaufmann y Gil-Aluja (1989) indican: "La introducción de una valuación matizada entre 0 y 1 permite hacer intervenir niveles de verdad en la noción de incidencia. (...) Valores de 0 a 1 (la llamada valuación endecadaria)" (p. 26). Por lo tanto, las opiniones de los expertos serán tratadas en una escala endecadaria como la siguiente: 


\section{Tabla 2}

Escala endecadaria

\section{Grado de presunción $\alpha$}

\begin{tabular}{cl}
\hline 1 & Máxima incidencia \\
\hline 0,9 & Muchísima incidencia \\
\hline 0,8 & Mucha incidencia \\
\hline 0,7 & Incidencia importante \\
\hline 0,6 & Bastante incidencia \\
\hline 0,5 & Tiene incidencia como no tiene incidencia \\
\hline 0,4 & Influente incidencia \\
\hline 0,3 & Algo de incidencia \\
\hline 0,2 & Poca incidencia \\
\hline 0,1 & Mínima incidencia \\
\hline 0 & No existe incidencia \\
\hline
\end{tabular}

Se procede a efectuar la consulta a 10 expertos del SRI, entre los que se encuentran especialistas, analistas, coordinadores de talento humano, así como a servidores administrativos que han participado en estos procesos de evaluación, tanto nacional como zonal, para que otorguen su criterio relacionado a la incidencia entre las acciones y efectos detallados en la tabla 1, como por ejemplo la incidencia de socializar el método, instrumentos y factores que intervienen en el proceso de evaluación de desempeño en la disminución de la resistencia a la evaluación, la misma que puede ser tanto del evaluador como del evaluado, y así para cada uno de los puntos de la matriz, obteniendo los siguientes resultados de la tabla 3 para el caso de "Socializar el métodos, instrumentos y factores de evaluación" con la incidencia en "Disminuir la resistencia a la evaluación": Incidencia de 1 . Socializar los métodos, instrumentos y factores de evaluación en A. Disminuir la resistencia a la evaluación 


\section{Tabla 3}

Opinión de los expertos

\begin{tabular}{cc} 
& $1 \mathrm{~A}$ \\
\hline Experto 1 & 1,0 \\
\hline Experto 2 & 1,0 \\
\hline Experto 3 & 1,0 \\
\hline Experto 4 & 1,0 \\
\hline Experto 5 & 0,8 \\
\hline Experto 6 & 1,0 \\
\hline Experto 7 & 0,6 \\
\hline Experto 8 & 0,9 \\
\hline Experto 9 & 1,0 \\
\hline Experto 10 & 0,6 \\
\hline
\end{tabular}

Con los datos presentados y volcados en la tabla anterior, se procede a determinar la frecuencia, es decir las repeticiones del grado de presunción con relación a la cantidad de expertos consultados por cada combinación de las posibles incidencias. Una vez obtenida la frecuencia, se procede a normalizar la serie, es decir, dividir el dato de la frecuencia para el número total de expertos, que, para este caso de estudio, son 10.

Posterior a esto, se acumula la serie de abajo hacia arriba, es decir, desde el último valor en forma ascendente hasta obtener el valor de la unidad, a partir de este valor, todos los valores se consideran como 1, para finalmente sumar estos resultados sin considerar to obtenido para 0 , tomando el ejemplo anterior, se detalla el resultado en la siguiente tabla:

\section{Tabla 4}

Normalizar y acumular la serie

\begin{tabular}{cccc}
$\begin{array}{c}\text { GRADO DE } \\
\text { PRESUNCIÓN } \boldsymbol{\alpha}\end{array}$ & FRECUENCIA & $\begin{array}{c}\text { NORMALIZACIÓN DE LA } \\
\text { SERIE }\end{array}$ & ACUMULACIÓN \\
\hline 0 & 0 & 0,00 & 1,00 \\
\hline 0,1 & 0 & 0,00 & 1,00 \\
\hline 0,2 & 0 & 0,00 & 1,00 \\
\hline 0,3 & 0 & 0,00 & 1,00 \\
\hline 0,4 & 0 & 0,00 & 1,00 \\
\hline
\end{tabular}




\section{CIENCIAMATRIA}

Revista Interdisciplinaria de Humanidades, Educación, Ciencia y Tecnología

Año V. Vol. V. NN1. Edición Especial. 2019

Hecho el depósito de ley: pp201602FA4721

ISSN-L: 2542-3029; ISSN: 2610-802X

Universidad Nacional Experimental Francisco de Miranda (UNEFM). Santa Ana de Coro. Venezuela

Edisson Antonio Carrión Agila; Juan Carlos Erazo Álvarez; Cecilia Ivonne Narváez Zurita; Diego Fernando Trelles Vicuña

\begin{tabular}{cccc}
\hline 0,5 & 0 & 0,00 & 1,00 \\
\hline 0,6 & 2 & 0,20 & 1,00 \\
\hline 0,7 & 0 & 0,00 & 0,80 \\
\hline 0,8 & 1 & 0,10 & 0,80 \\
\hline 0,9 & 1 & 0,10 & 0,70 \\
\hline 1 & 6 & 0,60 & 0,60 \\
\hline TOTALES & 10 & 1 & 8,90
\end{tabular}

Al valor que se obtiene de la sumatoria de las frecuencias acumuladas, se divide para el total de valores que fueron consideraos dentro del grado de presunción, es decir 10, que para el ejemplo es: $8,90 \div 10=0,89$. Esta operación se realiza para todas las acciones y su incidencia en los efectos, obteniendo los siguientes resultados:

Tabla 5

Matriz de incidencia

\begin{tabular}{|c|c|c|c|c|c|c|c|c|c|c|}
\hline & $\mathbf{A}$ & $\mathbf{B}$ & $\mathbf{C}$ & $\mathbf{D}$ & $\mathbf{E}$ & $\mathbf{F}$ & $\mathbf{G}$ & $\mathbf{H}$ & $\mathbf{I}$ & $\mathbf{J}$ \\
\hline $\mathbf{1}$ & $\mathbf{0 , 8 9}$ & 0,85 & 0,79 & 0,77 & 0,79 & 0,77 & 0,80 & 0,79 & 0,80 & 0,78 \\
\hline $\mathbf{2}$ & 0,86 & 0,89 & 0,85 & 0,84 & 0,85 & 0,85 & 0,87 & 0,85 & 0,82 & 0,83 \\
\hline $\mathbf{3}$ & 0,50 & 0,83 & 0,88 & 0,49 & 0,38 & 0,24 & 0,20 & 0,28 & 0,50 & 0,40 \\
\hline $\mathbf{4}$ & 0,63 & 0,51 & 0,73 & 0,80 & 0,62 & 0,55 & 0,58 & 0,59 & 0,73 & 0,84 \\
\hline $\mathbf{5}$ & 0,43 & 0,53 & 0,64 & 0,64 & 0,46 & 0,44 & 0,45 & 0,42 & 0,83 & 0,73 \\
\hline $\mathbf{6}$ & 0,85 & 0,85 & 0,82 & 0,78 & 0,86 & 0,87 & 0,87 & 0,88 & 0,87 & 0,79 \\
\hline $\mathbf{7}$ & 0,86 & 0,75 & 0,61 & 0,59 & 0,79 & 0,81 & 0,87 & 0,87 & 0,60 & 0,53 \\
\hline $\mathbf{8}$ & 0,59 & 0,30 & 0,71 & 0,53 & 0,24 & 0,25 & 0,26 & 0,30 & 0,44 & 0,27 \\
\hline $\mathbf{9}$ & 0,61 & 0,76 & 0,88 & 0,76 & 0,73 & 0,82 & 0,45 & 0,71 & 0,75 & 0,89 \\
\hline $\mathbf{1 0}$ & 0,75 & 0,87 & 0,86 & 0,80 & 0,78 & 0,77 & 0,79 & 0,78 & 0,70 & 0,86 \\
\hline
\end{tabular}


Siendo:

\begin{tabular}{|c|c|c|c|}
\hline 1 & $\begin{array}{l}\text { Socializar el métodos, instrumentos y } \\
\text { factores de evaluación }\end{array}$ & A & Disminuir la resistencia a la evaluación \\
\hline 2 & $\begin{array}{l}\text { Mantener actualizadas las metodologías y } \\
\text { los criterios de evaluación }\end{array}$ & $\mathrm{B}$ & $\begin{array}{l}\text { Elaborar un óptimo instrumento de } \\
\text { evaluación }\end{array}$ \\
\hline 3 & Infraestructura tecnológica actualizada & $\mathrm{C}$ & $\begin{array}{l}\text { Evitar inconvenientes operativos en la } \\
\text { ejecución del proceso de evaluación }\end{array}$ \\
\hline 4 & $\begin{array}{l}\text { Mantener entrevistas regulares de } \\
\text { avances en los procesos para el } \\
\text { cumplimiento de indicadores }\end{array}$ & D & $\begin{array}{l}\text { Evitar criterios de evaluación basados } \\
\text { en una sola impresión }\end{array}$ \\
\hline 5 & $\begin{array}{l}\text { Implementar una bitácora de } \\
\text { acontecimientos (negativos y positivos) } \\
\text { sobre el cumplimiento de indicadores }\end{array}$ & $\mathrm{E}$ & $\begin{array}{l}\text { Evitar criterios negativos del evaluador } \\
\text { por acontecimientos recientes }\end{array}$ \\
\hline 6 & $\begin{array}{l}\text { Establecer un mecanismo de } \\
\text { comunicación efectiva y avances de } \\
\text { resultados }\end{array}$ & $\mathrm{F}$ & $\begin{array}{l}\text { Evitar generar opiniones personales } \\
\text { hacia el evaluado como elementos } \\
\text { culturales }\end{array}$ \\
\hline 7 & $\begin{array}{l}\text { Implementar la aprobación del jefe } \\
\text { mediato en la evaluación del servidor }\end{array}$ & G & Disminuir la subjetividad del evaluador \\
\hline 8 & $\begin{array}{l}\text { Implementar una metodología de } \\
\text { levantamiento continuo de necesidades de } \\
\text { capacitación }\end{array}$ & $\mathrm{H}$ & $\begin{array}{l}\text { Detectar fallas en conocimientos que } \\
\text { impidan la consecución de objetivos y } \\
\text { la incompetencia del evaluado }\end{array}$ \\
\hline 9 & $\begin{array}{l}\text { Mantener actualizados los manuales y } \\
\text { descriptivos de puestos en donde se } \\
\text { definan claramente las competencias }\end{array}$ & 1 & $\begin{array}{l}\text { Definiciones claras de las competencias } \\
\text { necesarias para el cargo }\end{array}$ \\
\hline 10 & $\begin{array}{l}\text { Socializar los descriptores de cargos con } \\
\text { el personal }\end{array}$ & $\mathrm{J}$ & $\begin{array}{l}\text { Posibilidad de identificar mejoras en el } \\
\text { desarrollo de competencias }\end{array}$ \\
\hline
\end{tabular}

Tomando como base la tabla 5 de matriz de incidencia, aplicamos la operación de la teoría de efectos olvidados, con la finalidad de determinar las variables escondidas $u$ omitidas por los servidores de la Institución que ejecutan o están inmersos en la ejecución del proceso de evaluación de desempeño, entre la incidencia acción/efecto. Para el caso de esta investigación, se construyó una matriz cuadrada, dado que el número de elementos en las columnas (Acciones) es el mismo que el de las filas (Efectos).

Para proceder con los cálculos, el primer paso es convulsionar la matriz, esto es, determinar el número mayor de una serie de números menores, los mismos que resultan 


\section{CIENCIAMATRIA \\ Revista Interdisciplinaria de Humanidades, Educación, Ciencia y Tecnología \\ Año V. Vol. V. NN1. Edición Especial. 2019 \\ Hecho el depósito de ley: pp201602FA4721 \\ ISSN-L: 2542-3029; ISSN: 2610-802X \\ Universidad Nacional Experimental Francisco de Miranda (UNEFM). Santa Ana de Coro. Venezuela}

Edisson Antonio Carrión Agila; Juan Carlos Erazo Álvarez; Cecilia Ivonne Narváez Zurita; Diego Fernando Trelles Vicuña

de la comparación entre las filas con las columnas de la matriz de incidencia, por lo tanto, se convulsiona la matriz por sí misma, y de este proceso se obtiene la matriz II. Para entenderlo de mejor manera, se procede a realizar lo indicado tomando como ejemplo la fila 1 con la columna "A", entonces:

\begin{tabular}{|c|c|c|c|c|c|c|c|c|c|c|}
\hline & $\mathbf{A}$ & $\mathbf{B}$ & $\mathbf{C}$ & $\mathbf{D}$ & $\mathbf{E}$ & $\mathbf{F}$ & $\mathbf{G}$ & $\mathbf{H}$ & $\mathbf{I}$ & $\mathbf{J}$ \\
\hline $\mathbf{1}$ & $\mathbf{0 , 8 9}$ & 0,85 & 0,79 & 0,77 & 0,79 & 0,77 & 0,80 & 0,79 & 0,80 & 0,78 \\
\hline $\mathbf{2}$ & 0,86 & 0,89 & 0,85 & 0,84 & 0,85 & 0,85 & 0,87 & 0,85 & 0,82 & 0,83 \\
\hline $\mathbf{3}$ & 0,50 & 0,83 & 0,88 & 0,49 & 0,38 & 0,24 & 0,20 & 0,28 & 0,50 & 0,40 \\
\hline $\mathbf{4}$ & 0,63 & 0,51 & 0,73 & 0,80 & 0,62 & 0,55 & 0,58 & 0,59 & 0,73 & 0,84 \\
\hline $\mathbf{5}$ & 0,43 & 0,53 & 0,64 & 0,64 & 0,46 & 0,44 & 0,45 & 0,42 & 0,83 & 0,73 \\
\hline $\mathbf{6}$ & 0,85 & 0,85 & 0,82 & 0,78 & 0,86 & 0,87 & 0,87 & 0,88 & 0,87 & 0,79 \\
\hline $\mathbf{7}$ & 0,86 & 0,75 & 0,61 & 0,59 & 0,79 & 0,81 & 0,87 & 0,87 & 0,60 & 0,53 \\
\hline $\mathbf{8}$ & 0,59 & 0,30 & 0,71 & 0,53 & 0,24 & 0,25 & 0,26 & 0,30 & 0,44 & 0,27 \\
\hline $\mathbf{9}$ & 0,61 & 0,76 & 0,88 & 0,76 & 0,73 & 0,82 & 0,45 & 0,71 & 0,75 & 0,89 \\
\hline $\mathbf{1 0}$ & 0,75 & 0,87 & 0,86 & 0,80 & 0,78 & 0,77 & 0,79 & 0,78 & 0,70 & 0,86 \\
\hline
\end{tabular}

\begin{tabular}{|c|c|c|c|c|c|c|c|c|c|c|}
\hline & $\mathbf{A}$ & $\mathbf{B}$ & $\mathbf{C}$ & $\mathbf{D}$ & $\mathbf{E}$ & $\mathbf{F}$ & $\mathbf{G}$ & $\mathbf{H}$ & $\mathbf{I}$ & $\mathbf{J}$ \\
\hline $\mathbf{1}$ & 0,89 & 0,85 & 0,79 & 0,77 & 0,79 & 0,77 & 0,80 & 0,79 & 0,80 & 0,78 \\
\hline $\mathbf{2}$ & 0,86 & 0,89 & 0,85 & 0,84 & 0,85 & 0,85 & 0,87 & 0,85 & 0,82 & 0,83 \\
\hline $\mathbf{3}$ & 0,50 & 0,83 & 0,88 & 0,49 & 0,38 & 0,24 & 0,20 & 0,28 & 0,50 & 0,40 \\
\hline $\mathbf{4}$ & 0,63 & 0,51 & 0,73 & 0,80 & 0,62 & 0,55 & 0,58 & 0,59 & 0,73 & 0,84 \\
\hline $\mathbf{5}$ & 0,43 & 0,53 & 0,64 & 0,64 & 0,46 & 0,44 & 0,45 & 0,42 & 0,83 & 0,73 \\
\hline $\mathbf{6}$ & 0,85 & 0,85 & 0,82 & 0,78 & 0,86 & 0,87 & 0,87 & 0,88 & 0,87 & 0,79 \\
\hline $\mathbf{7}$ & 0,86 & 0,75 & 0,61 & 0,59 & 0,79 & 0,81 & 0,87 & 0,87 & 0,60 & 0,53 \\
\hline $\mathbf{8}$ & 0,59 & 0,30 & 0,71 & 0,53 & 0,24 & 0,25 & 0,26 & 0,30 & 0,44 & 0,27 \\
\hline $\mathbf{9}$ & 0,61 & 0,76 & 0,88 & 0,76 & 0,73 & 0,82 & 0,45 & 0,71 & 0,75 & 0,89 \\
\hline $\mathbf{1 0}$ & 0,75 & 0,87 & 0,86 & 0,80 & 0,78 & 0,77 & 0,79 & 0,78 & 0,70 & 0,86 \\
\hline
\end{tabular}

$(A 1 \vee A 1) \wedge(B 1 \vee A 2) \wedge(C 1 \vee A 3) \wedge(D 1 \vee A 4) \wedge(E 1 \vee A 5) \wedge(F 1 \vee A 6) \wedge(G 1 \vee A 7)$

ᄉ $(H 1 \vee A 8) \wedge(I 1 \vee A 9) \wedge(J 1 \vee A 10)$

$(0,89 \vee 0,89) \wedge(0,85 \vee 0,86) \wedge(0,79 \vee 0,50) \wedge(0,77 \vee 0,63) \wedge(0,79 \vee 0,43) \wedge(0,77 \curlyvee 0,85)$

ᄉ $(0,80 \vee 0,86) \wedge(0,79 \vee 0,59) \wedge(0,80 \vee 0,61) \wedge(0,78 \vee 0,75)$

$\underline{0,89 \wedge 0,85 \wedge 0,50 \wedge 0,63 \wedge 0,43 \wedge 0,77 \wedge 0,80 \wedge 0,59 \wedge 0,61 \wedge 0,75=\mathbf{0 , 8 9}}$

Se puede observar que, de todos los valores menores de cada intervalo, se selecciona el mayor, que para el caso es de 0,89. Este valor se coloca en la celda A1 de la matriz II, y se efectúa el mismo procedimiento para los demás intervalos de la primera matriz, componiendo así la matriz II, que llega a ser la matriz convolucionada. Como resultado, se presenta la matriz II 
CIENCIAMATRIA

Revista Interdisciplinaria de Humanidades, Educación, Ciencia y Tecnología

Año V. Vol. V. №1. Edición Especial. 2019

Hecho el depósito de ley: pp201602FA4721

ISSN-L: 2542-3029; ISSN: 2610-802X

Universidad Nacional Experimental Francisco de Miranda (UNEFM). Santa Ana de Coro. Venezuela

Edisson Antonio Carrión Agila; Juan Carlos Erazo Álvarez; Cecilia Ivonne Narváez Zurita; Diego Fernando Trelles Vicuña

Tabla 6

Matriz convolucionada

\begin{tabular}{|r|c|c|c|c|c|c|c|c|c|c|}
\hline \multicolumn{1}{|c|}{ II } & A & B & C & D & E & F & G & H & I & J \\
\hline $\mathbf{1}$ & 0,89 & 0,85 & 0,85 & 0,84 & 0,85 & 0,85 & 0,85 & 0,85 & 0,82 & 0,83 \\
\hline $\mathbf{2}$ & 0,86 & 0,89 & 0,85 & 0,84 & 0,85 & 0,85 & 0,87 & 0,87 & 0,85 & 0,84 \\
\hline $\mathbf{3}$ & 0,83 & 0,83 & 0,88 & 0,83 & 0,83 & 0,83 & 0,83 & 0,83 & 0,82 & 0,83 \\
\hline $\mathbf{4}$ & 0,75 & 0,84 & 0,84 & 0,80 & 0,78 & 0,77 & 0,79 & 0,78 & 0,73 & 0,84 \\
\hline $\mathbf{5}$ & 0,73 & 0,76 & 0,83 & 0,76 & 0,73 & 0,82 & 0,73 & 0,73 & 0,75 & 0,83 \\
\hline $\mathbf{6}$ & 0,86 & 0,85 & 0,87 & 0,84 & 0,86 & 0,87 & 0,87 & 0,87 & 0,87 & 0,87 \\
\hline $\mathbf{7}$ & 0,86 & 0,85 & 0,81 & 0,78 & 0,81 & 0,81 & 0,87 & 0,87 & 0,81 & 0,79 \\
\hline $\mathbf{8}$ & 0,59 & 0,71 & 0,71 & 0,59 & 0,59 & 0,59 & 0,59 & 0,59 & 0,59 & 0,59 \\
\hline $\mathbf{9}$ & 0,82 & 0,87 & 0,88 & 0,80 & 0,82 & 0,82 & 0,82 & 0,82 & 0,82 & 0,86 \\
\hline $\mathbf{1 0}$ & 0,86 & 0,87 & 0,86 & 0,84 & 0,85 & 0,85 & 0,87 & 0,85 & 0,82 & 0,86 \\
\hline
\end{tabular}

Los datos presentados en la matriz II, corresponden a los efectos olvidados de primera generación, sin embargo, es necesario continuar con los cálculos para determinar la variable escondida, para el efecto, restamos los valores de la matriz I con los de la matriz II respetando los cuadrantes, dicho resultado se expresa en valor absoluto y componen la matriz III:

\section{Tabla 7}

Resta de Matriz I y II

\begin{tabular}{|c|c|c|c|c|c|c|c|c|c|c|}
\hline II & $\mathbf{A}$ & $\mathbf{B}$ & $\mathbf{C}$ & $\mathbf{D}$ & $\mathbf{E}$ & $\mathbf{F}$ & $\mathbf{G}$ & $\mathbf{H}$ & $\mathbf{I}$ & $\mathbf{J}$ \\
\hline $\mathbf{1}$ & 0,89 & 0,85 & 0,85 & 0,84 & 0,85 & 0,85 & 0,85 & 0,85 & 0,82 & 0,83 \\
\hline $\mathbf{2}$ & 0,86 & 0,89 & 0,85 & 0,84 & 0,85 & 0,85 & 0,87 & 0,87 & 0,85 & 0,84 \\
\hline $\mathbf{3}$ & 0,83 & 0,83 & 0,88 & 0,83 & 0,83 & 0,83 & 0,83 & 0,83 & 0,82 & 0,83 \\
\hline $\mathbf{4}$ & 0,75 & 0,84 & 0,84 & 0,80 & 0,78 & 0,77 & 0,79 & 0,78 & 0,73 & 0,84 \\
\hline $\mathbf{5}$ & 0,73 & 0,76 & 0,83 & 0,76 & 0,73 & 0,82 & 0,73 & 0,73 & 0,75 & 0,83 \\
\hline $\mathbf{6}$ & 0,86 & 0,85 & 0,87 & 0,84 & 0,86 & 0,87 & 0,87 & 0,87 & 0,87 & 0,87 \\
\hline $\mathbf{7}$ & 0,86 & 0,85 & 0,81 & 0,78 & 0,81 & 0,81 & 0,87 & 0,87 & 0,81 & 0,79 \\
\hline $\mathbf{8}$ & 0,59 & 0,71 & 0,71 & 0,59 & 0,59 & 0,59 & 0,59 & 0,59 & 0,59 & 0,59 \\
\hline $\mathbf{9}$ & 0,82 & 0,87 & 0,88 & 0,80 & 0,82 & 0,82 & 0,82 & 0,82 & 0,82 & 0,86 \\
\hline $\mathbf{1 0}$ & 0,86 & 0,87 & 0,86 & 0,84 & 0,85 & 0,85 & 0,87 & 0,85 & 0,82 & 0,86 \\
\hline
\end{tabular}

$(-)$

\begin{tabular}{|c|c|c|c|c|c|c|c|c|c|c|}
\hline & $\mathbf{A}$ & $\mathbf{B}$ & $\mathbf{C}$ & $\mathbf{D}$ & $\mathbf{E}$ & $\mathbf{F}$ & $\mathbf{G}$ & $\mathbf{H}$ & $\mathbf{I}$ & $\mathbf{J}$ \\
\hline $\mathbf{1}$ & $\mathbf{0 , 8 9}$ & 0,85 & 0,79 & 0,77 & 0,79 & 0,77 & 0,80 & 0,79 & 0,80 & 0,78 \\
\hline $\mathbf{2}$ & 0,86 & 0,89 & 0,85 & 0,84 & 0,85 & 0,85 & 0,87 & 0,85 & 0,82 & 0,83 \\
\hline
\end{tabular}




\section{CIENCIAMATRIA}

Revista Interdisciplinaria de Humanidades, Educación, Ciencia y Tecnología

Año V. Vol. V. NN1. Edición Especial. 2019

Hecho el depósito de ley: pp201602FA4721

ISSN-L: 2542-3029; ISSN: 2610-802X

Universidad Nacional Experimental Francisco de Miranda (UNEFM). Santa Ana de Coro. Venezuela

Edisson Antonio Carrión Agila; Juan Carlos Erazo Álvarez; Cecilia Ivonne Narváez Zurita; Diego Fernando Trelles Vicuña

\begin{tabular}{|c|c|c|c|c|c|c|c|c|c|c|}
$\mathbf{3}$ & 0,50 & 0,83 & 0,88 & 0,49 & 0,38 & 0,24 & 0,20 & 0,28 & 0,50 & 0,40 \\
\hline $\mathbf{4}$ & 0,63 & 0,51 & 0,73 & 0,80 & 0,62 & 0,55 & 0,58 & 0,59 & 0,73 & 0,84 \\
\hline $\mathbf{5}$ & 0,43 & 0,53 & 0,64 & 0,64 & 0,46 & 0,44 & 0,45 & 0,42 & 0,83 & 0,73 \\
\hline $\mathbf{6}$ & 0,85 & 0,85 & 0,82 & 0,78 & 0,86 & 0,87 & 0,87 & 0,88 & 0,87 & 0,79 \\
\hline $\mathbf{7}$ & 0,86 & 0,75 & 0,61 & 0,59 & 0,79 & 0,81 & 0,87 & 0,87 & 0,60 & 0,53 \\
\hline $\mathbf{8}$ & 0,59 & 0,30 & 0,71 & 0,53 & 0,24 & 0,25 & 0,26 & 0,30 & 0,44 & 0,27 \\
\hline $\mathbf{9}$ & 0,61 & 0,76 & 0,88 & 0,76 & 0,73 & 0,82 & 0,45 & 0,71 & 0,75 & 0,89 \\
\hline $\mathbf{1 0}$ & 0,75 & 0,87 & 0,86 & 0,80 & 0,78 & 0,77 & 0,79 & 0,78 & 0,70 & 0,86 \\
\hline
\end{tabular}

$(=)$

Tabla 8

Matriz de efectos olvidados

\begin{tabular}{|c|c|c|c|c|c|c|c|c|c|c|}
\hline III & A & B & C & D & E & F & G & H & I & J \\
\hline $\mathbf{1}$ & 0,00 & 0,00 & 0,06 & 0,07 & 0,06 & 0,08 & 0,05 & 0,06 & 0,02 & 0,05 \\
\hline $\mathbf{2}$ & 0,00 & 0,00 & 0,00 & 0,00 & 0,00 & 0,00 & 0,00 & 0,02 & 0,03 & 0,01 \\
\hline $\mathbf{3}$ & 0,33 & 0,00 & 0,00 & 0,34 & 0,45 & 0,59 & 0,63 & 0,55 & 0,32 & 0,43 \\
\hline $\mathbf{4}$ & 0,12 & 0,33 & 0,11 & 0,00 & 0,16 & 0,22 & 0,21 & 0,19 & 0,00 & 0,00 \\
\hline $\mathbf{5}$ & 0,30 & 0,23 & 0,19 & 0,12 & 0,27 & 0,38 & 0,28 & 0,31 & 0,08 & 0,10 \\
\hline $\mathbf{6}$ & 0,01 & 0,00 & 0,05 & 0,06 & 0,00 & 0,00 & 0,00 & 0,01 & 0,00 & 0,08 \\
\hline $\mathbf{7}$ & 0,00 & 0,10 & 0,20 & 0,19 & 0,02 & 0,00 & 0,00 & 0,00 & 0,21 & 0,26 \\
\hline $\mathbf{8}$ & 0,00 & 0,41 & 0,00 & 0,06 & 0,35 & 0,34 & 0,33 & 0,29 & 0,15 & 0,32 \\
\hline $\mathbf{9}$ & 0,21 & 0,11 & 0,00 & 0,04 & 0,09 & 0,00 & 0,37 & 0,11 & 0,07 & 0,03 \\
\hline $\mathbf{1 0}$ & 0,11 & 0,00 & 0,00 & 0,04 & 0,07 & 0,08 & 0,08 & 0,07 & 0,12 & 0,00 \\
\hline
\end{tabular}

Para determinar las variables escondidas o efectos olvidados, se basa en los valores obtenidos en la matriz de la tabla 8 (Matriz que contiene efectos olvidados), se escoge los valores más cercanos a la unidad, en el caso de esta investigación se consideran los valores " $\alpha$ " superiores a 0,6 ubicados en las coordenadas $(3, G)$, estos valores son analizados de manera cómo la acción incide sobre el efecto, encontrando el efecto olvidado de incidencia de causalidad entre estas dos variables. Para el efecto, se traslada en el mismo orden a la matriz original "I" (Tabla 5. Matriz de incidencia), con el propósito de hallar el efecto olvidado, y con el fin de minimizar el procedimiento en este punto, 


\section{CIENCIAMATRIA}

Revista Interdisciplinaria de Humanidades, Educación, Ciencia y Tecnología

Año V. Vol. V. №1. Edición Especial. 2019

Hecho el depósito de ley: pp201602FA4721

ISSN-L: 2542-3029; ISSN: 2610-802X

Universidad Nacional Experimental Francisco de Miranda (UNEFM). Santa Ana de Coro. Venezuela

Edisson Antonio Carrión Agila; Juan Carlos Erazo Álvarez; Cecilia Ivonne Narváez Zurita; Diego Fernando Trelles Vicuña

procedemos a sumar los valores entre acción y efecto, con el fin de determinar el valor mayor, el mismo que representa el efecto olvidado, tal como se muestra en la siguiente tabla.

\section{Tabla 9}

Matriz de efectos olvidados

\begin{tabular}{ccccc}
\multicolumn{3}{c}{3} & & $\mathrm{H}$ \\
\hline $\mathrm{A}$ & 0,50 & 1,29 & $\mathrm{~A}$ & 0,79 \\
\hline $\mathrm{B}$ & 0,83 & $\mathbf{1 , 6 8}$ & $\mathrm{B}$ & 0,85 \\
\hline $\mathrm{C}$ & 0,88 & 1,16 & $\mathrm{C}$ & 0,28 \\
\hline $\mathrm{D}$ & 0,49 & 1,08 & $\mathrm{D}$ & 0,59 \\
\hline $\mathrm{E}$ & 0,38 & 0,80 & $\mathrm{E}$ & 0,42 \\
\hline $\mathrm{F}$ & 0,24 & 1,12 & $\mathrm{~F}$ & 0,88 \\
\hline $\mathrm{G}$ & 0,20 & 1,07 & $\mathrm{G}$ & 0,87 \\
\hline $\mathrm{H}$ & 0,28 & 0,58 & $\mathrm{H}$ & 0,30 \\
\hline $\mathrm{I}$ & 0,50 & 1,21 & $\mathrm{I}$ & 0,71 \\
\hline $\mathrm{J}$ & 0,40 & 1,18 & $\mathrm{~J}$ & 0,78 \\
\hline
\end{tabular}

Como resultado del procedimiento anteriormente descrito, y con el fin de que se entienda de mejor manera, se representa el resultado en el siguiente gráfico. 


\section{CIENCIAMATRIA}

Revista Interdisciplinaria de Humanidades, Educación, Ciencia y Tecnología

Año V. Vol. V. N¹. Edición Especial. 2019

Hecho el depósito de ley: pp201602FA4721

ISSN-L: 2542-3029; ISSN: 2610-802X

Universidad Nacional Experimental Francisco de Miranda (UNEFM). Santa Ana de Coro. Venezuela

Edisson Antonio Carrión Agila; Juan Carlos Erazo Álvarez; Cecilia Ivonne Narváez Zurita; Diego Fernando Trelles Vicuña

\section{Figura 2}

Efecto olvidado
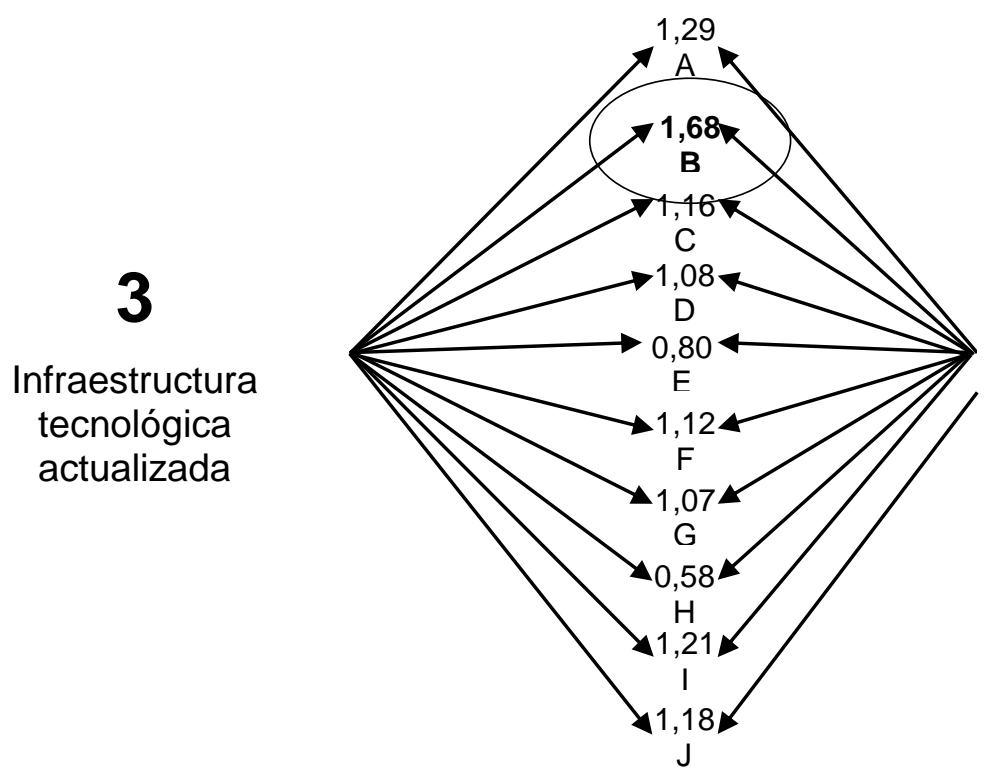

G

Disminuir la subjetividad del evaluador

De la metodología aplicada, y utilizando la herramienta de avanzada que brinda la lógica difusa, se obtuvo como resultado una infraestructura tecnológica actualizada incide en gran medida en coadyuvar en la disminución de la subjetividad del evaluador, a través de la variable omitida de elaborar un óptimo instrumento de evaluación.

Determinado el efecto omitido, se propone dicha variable a los ejecutores del proceso de evaluación de desempeño, para la mejora en este proceso y dar solución problema de esta investigación.

\section{CONCLUSIONES}

El uso de la lógica difusa en los procesos de evaluación tiene una gran trascendencia en los procesos de evaluación de desempeño, que, para el caso de esta investigación, a través del uso de la herramienta de la teoría de los efectos olvidados, se determinó cual es la variable omitida y que no es considerada de mayor trascendencia en el proceso. 
Si bien como se mencionó al inicio, las instituciones públicas deben enmarcarse en lo que la norma dictamina, es posible implementar estas metodologías en procedimientos internos para el cumplimiento de la normativa.

Dichos procedimientos deben implementar un formato propio del instrumento de evaluación, considerando lo obtenido de la aplicación de la matriz de efectos olvidados. Por lo tanto, se recomienda elaborar un instrumento propio de la institución que permita abarcar elementos referentes a las competencias necesarias para el cargo, dado que este factor o variable está en tercer lugar en la matriz de efectos olvidados, lo que a su vez permite disminuir la resistencia a la evaluación, así como la identificación de mejoras en el desarrollo de las competencias de los servidores.

Finalmente, otro elemento con una incidencia fuerte de acuerdo a los resultados obtenidos, es la opinión personal del evaluado, el mismo que se abarca en la subjetividad del evaluador, la misma que se vería ampliamente reducida con un instrumento adecuado, así como permite una retroalimentación efectiva durante todo el proceso.

\section{REFERENCIAS CONSULTADAS}

1. Alles, M. (2008). Desempeño por competencias: Evaluacion 360. Buenos Aires, Argentina: Granica.

2. Alles, M. (2013). Dirección Estratégica de Recursos Humanos. Gestión por competencias. Buenos Aires, Argentina: Granica.

3. Asamblea Nacional Constituyente. (2010). Ley orgánica del servicio público (LOSEP). Quito: Registro Oficial Suplemento 294 de 06-oct.-2010.

4. Cerón Miranda, M. G., Erazo Álvarez, J. C., Ormaza Andrade, J. E., \& Narváez Zurita, C. I. (2019). La remuneración variable como eje estratégico de la gestión de Talento Humano en la Unidad de Negocio Hidropaute. Visionario Digital, 7-27.

5. Cordero, J., Narváez, C., \& Erazo, J. (2019). La evaluación por competencias: una herramienta para determinar la productividad del talento humano. Digital Publisher, 76-96. 


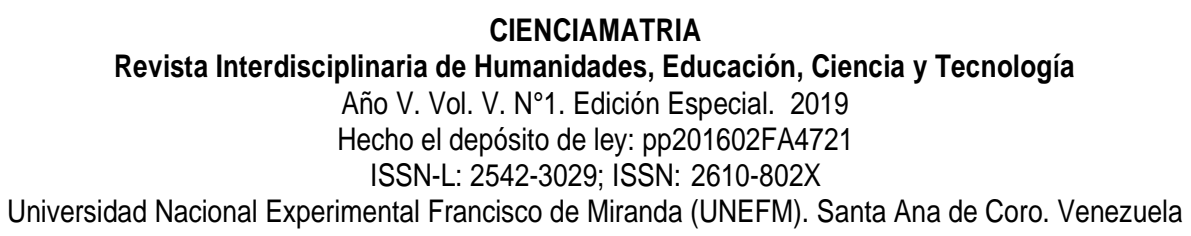

Edisson Antonio Carrión Agila; Juan Carlos Erazo Álvarez; Cecilia Ivonne Narváez Zurita; Diego Fernando Trelles Vicuña

6. Gento, A., Lazzari, L. L., \& Machado, E. A. (2001). Reflexiones acerca de las matrices de incidencia y la recuperación de efectos olvidados. Cuadernos del CIMBAGE, 11-27.

7. Gil, A., \& Barcellos, L. (2010). UNA APLICACIÓN DE LA METODOLOGÍA DE LOS EFECTOS OLVIDADOS: LOS FACTORES QUE CONTRIBUYEN AL CRECIMIENTO SOSTENIBLE DE LA EMPRESA. Cuadernos del CIMBAGE, 2334. Obtenido de http://www.redalyc.org/articulo.oa?id=46213329002

8. Kaufmann, A., \& Gil, J. (1989). Modelos para la investigación de efectos olvidados. Barcelona, España: Milladoiro.

9. Kaufmann, A., \& Gil, J. (1993). Técnicas especiales para la gestión de expertos. Santiago de Compostela, España: Milladoiro.

10. Luna Altamirano, K., Tinto Arandes, J., Sarmiento Espinoza, W., \& Cisneros Quintanilla, D. (2017). TRATAMIENTO DE IMPAGOS BAJO EL ENFOQUE DE LA INCERTIDUMBRE CON LA APLICACIÓN DE REDES NEURONALES (CASO ARTESANOS DE CALZADO CANTÓN GUALACEO PROVINCIA DEL AZUAY). Ciencias Pedagógicas e Innovación.

11. Manjarrés, A., Castell, R., \& Luna, C. (2013). Modelo de Evaluación del Desempeño Basado en Competencias. INGENIARE, 11-29.

12. Ministerio del Trabajo. (2018). Acuerdo Ministerial N MDT-2018-0041. Quito: Suplemento al Registro Oficial No. 218.

13. Ministerio del Trabajo. (2019). Acuerdo ministerial nro. MDT-2019-022. Quito: Registro Oficial No. 437.

14. Payán, L., \& Luhrs, M. (2013). Evaluación de programas públicos en el marco de la realidad social. Metodología basada en la lógica difusa como sociales. Latinoamericana de Metodología de la Investigación Social, 8-23.

15. Pérez, I., \& León, B. (2007). Lógica difusa para principiantes. Teoría y práctica. Caracas: UCAB.

16. Rico F., M. A., \& Tinto A., J. (2010). Herramientas con base en subconjuntos borrosos. Propuesta procedimental para aplicar expertizaje y recuperar efectos olvidados en la información contable. Actualidad Contable Faces, 127-146. 
17. Rico F., M. A., \& Tinto Arandes, J. (2009). Matemática borrosa: algunas aplicaciones en las ciencias económicas, administrativas y contables. Contaduría Universidad de Antioquia, 199-214.

18. Servicio de Rentas Internas. (2016). Resolución NAC-DGERCGC16-00000383. Quito.

19. Servicio de Rentas Internas. (s.f.). sri.gob.ec. Obtenido de http://www.sri.gob.ec/web/guest/que-es-el-sri

20.Soler, R., \& Castillo, A. (2009). Competencia en la Incertidumbre. Contribuciones a la Economía.

21. Tinto Arandes, J., Luna Altamirano, K. A., \& Cisneros Quintanilla, D. P. (2017). Teoría de efectos olvidados en el rescate de la imagen comercial de los artesanos del calzado del cantón Gualaceo Provincia del Azuay. Visión Gerencial, 24-42.

22. Transparency International. (s.f.). transparency.org. Obtenido de https://www.transparency.org/cpi2018

23. Vidal Beltrán, K., Erazo Álvarez, J., \& Narváez Zurita, C. (2019). La lógica difusa como herramienta de evaluación financiera de proyectos de inversión. Revista Arbitrada Interdisciplinaria Koinonía, 4(1), 309-348. doi:http://dx.doi.org/10.35381/r.k.v4i1.460

24.Zadeh, L. A. (1965). Fuzzy sets. Information and control. The Journal of Symbolic Logic, 338-356.

\section{CONSULTED REFERENCES}

1. Alles, M. (2008). Performance by competences: 360 Evaluation. Buenos Aires, Argentina: Granica.

2. Alles, M. (2013). Strategic Directorate of Human Resources. Management by competencies. Buenos Aires, Argentina: Granica.

3. National Constituent Assembly. (2010). Organic Law of Public Service (LOSEP). Quito: Official Supplement Register 294 of Oct 6, 2010. 
4. Cerón Miranda, M. G., Erazo Álvarez, J. C., Ormaza Andrade, J. E., \& Narváez Zurita, C. I. (2019). Variable remuneration as a strategic axis of Human Talent management in the Hidropaute Business Unit. Digital Visionary, 7-27.

5. Cordero, J., Narváez, C., \& Erazo, J. (2019). Competency assessment: a tool to determine the productivity of human talent. Digital Publisher, 76-96.

6. Gento, A., Lazzari, L. L., \& Machado, E. A. (2001). Reflections on the incidence matrices and the recovery of forgotten effects. CIMBAGE Notebooks, 11-27.

7. Gil, A., \& Barcellos, L. (2010). AN APPLICATION OF THE METHODOLOGY OF FORGOTTEN EFFECTS: THE FACTORS THAT CONTRIBUTE TO THE SUSTAINABLE GROWTH OF THE COMPANY. CIMBAGE Notebooks, 23-34. Retrieved from http://www.redalyc.org/articulo.oa?id=46213329002

8. Kaufmann, A., \& Gil, J. (1989). Models for the investigation of forgotten effects. Barcelona, Spain: Milladoiro.

9. Kaufmann, A., \& Gil, J. (1993). Special techniques for expert management. Santiago de Compostela, Spain: Milladoiro.

10.Luna Altamirano, K., Tinto Arandes, J., Sarmiento Espinoza, W., \& Cisneros Quintanilla, D. (2017). TREATMENT OF PAYMENTS UNDER THE APPROACH TO UNCERTAINTY WITH THE APPLICATION OF NEURONAL NETWORKS (CASES OF CALZADO CANTÓN GUALACEO PROVINCE OF AZUAY). Pedagogical Sciences and Innovation.

11. Manjarrés, A., Castell, R., \& Luna, C. (2013). Competency Based Performance Evaluation Model. INGENIARE, 11-29.

12. Ministry of Labor. (2018). Ministerial Agreement No. MDT-2018-0041. Quito: Supplement to Official Registry No. 218.

13. Ministry of Labor. (2019). Ministerial Agreement no. MDT-2019-022. Quito: Official Registry No. 437.

14. Payán, L., \& Luhrs, M. (2013). Evaluation of public programs within the framework of social reality. Methodology based on fuzzy logic as social. Latin American Methodology of Social Research, 8-23. 


\section{CIENCIAMATRIA}

Revista Interdisciplinaria de Humanidades, Educación, Ciencia y Tecnología

Año V. Vol. V. NN1. Edición Especial. 2019

Hecho el depósito de ley: pp201602FA4721

ISSN-L: 2542-3029; ISSN: 2610-802X

Universidad Nacional Experimental Francisco de Miranda (UNEFM). Santa Ana de Coro. Venezuela

Edisson Antonio Carrión Agila; Juan Carlos Erazo Álvarez; Cecilia Ivonne Narváez Zurita; Diego Fernando Trelles Vicuña

15.Pérez, I., \& León, B. (2007). Fuzzy logic for beginners. Theory and practice. Caracas: UCAB.

16. Rico F., M. A., \& Tinto A., J. (2010). Tools based on fuzzy subsets. Procedural proposal to apply expertise and recover forgotten effects on accounting information. Accounting Faces, 127-146.

17. Rico F., M. A., \& Tinto Arandes, J. (2009). Blurred mathematics: some applications in economic, administrative and accounting sciences. Accounting University of Antioquia, 199-214.

18. Internal Revenue Service. (2016). Resolution NAC-DGERCGC16-00000383. Quito

19. Internal Revenue Service. (s.f.). sri.gob.ec. Retrieved from http://www.sri.gob.ec/web/guest/que-es-el-sri

20.Soler, R., \& Castillo, A. (2009). Uncertainty Competition. Contributions to the Economy.

21. Tinto Arandes, J., Luna Altamirano, K. A., \& Cisneros Quintanilla, D. P. (2017). Theory of forgotten effects in the rescue of the commercial image of the footwear craftsmen of the Gualaceo canton in the Province of Azuay. Management Vision, 24-42.

22. Transparency International. (s.f.). transparency.org. Obtained from https://www.transparency.org/cpi2018

23. Vidal Beltrán, K., Erazo Álvarez, J., \& Narváez Zurita, C. (2019). Diffuse logic as a financial evaluation tool for investment projects. Interdisciplinary Arbitrated Review Koinonía, 4 (1), 309-348. doi: http: //dx.doi.org/10.35381/r.k.v4i1.460

24.Zadeh, L.A. (1965). Fuzzy sets Information and control. The Journal of Symbolic Logic, 338-356.

(C2019 por los autores. Este artículo es de acceso abierto y distribuido según los términos y condiciones de la licencia Creative Commons Atribución-NoComercial-Compartirlgual 4.0 Internacional (CC BY-NC-

SA 4.0) (https://creativecommons.org/licenses/by-nc-sa/4.0/). 